\title{
cosmetics
}

ISSN 2079-9284

www.mdpi.com/journal/cosmetics

Review

\section{Determination of the Antioxidant Status of the Skin by In Vivo-Electron Paramagnetic Resonance (EPR) Spectroscopy}

\section{Silke Barbara Lohan 1, Anna-Christina Lauer ${ }^{1}$, Sophia Arndt ${ }^{1}$, Annette Friedrich 1,} Kathrin Tscherch ${ }^{2}$, Stefan F. Haag ${ }^{1}$, Maxim E. Darvin ${ }^{1}$, Henning Vollert ${ }^{3}$, Anke Kleemann ${ }^{4}$, Ingo Gersonde ${ }^{5}$, Norbert Groth ${ }^{6}$, Jürgen Lademann ${ }^{1}$, Sascha Rohn ${ }^{7}$ and Martina Claudia Meinke ${ }^{1, *}$

1 Department of Dermatology, Center of Experimental and Applied Cutaneous Physiology, Charité-Universitätsmedizin, Berlin 10117, Germany; E-Mails: silke.lohan@charite.de (S.B.L.); anna-christ.lauer@charite.de (A.C.L.); sophia.arndt@charite.de (S.A.);

Annette.Friedrich@charite.de (A.F.); stefan.haag78@googlemail.com (S.F.H.); maxim.darvin@charite.de (M.E.D.); juergen.lademann@charite.de (J.L.)

2 The JM Smucker Company, Orrville 44677, OH, USA; E-Mail: tscherch@gmail.com

3 BioActive Food GmbH, Bad Segeberg 23795, Germany; E-Mail: dr.vollert@bioactive-food.com

4 Klosterfrau Berlin GmbH, Berlin 10117, Germany; E-Mail: Anke.Kleemann@cassella-med.de

5 Laser-und Medizin-Technologie GmbH, Berlin 10117, Germany; E-Mail: i.gersonde@lmtb.de

6 Privatinstitut Galenus GmbH, Berlin 10117, German; E-Mail: info@galenus.com

7 Institute of Food Chemistry, Hamburg School of Food Science, University of Hamburg, Hamburg 20146, Germany; E-Mail: rohn@chemie.uni-hamburg.de

* Author to whom correspondence should be addressed: E-Mail: martina.meinke@charite.de; Tel.: +49-30-450-518-244.

Academic Editor: Enzo Berardesca

Received: 15 July 2015 / Accepted: 10 August 2015 / Published: 19 August 2015

\begin{abstract}
Organisms produce free radicals which are essential for various metabolic processes (enzymatic oxidation, cellular respiration, signaling). Antioxidants are important chemical compounds that specifically prevent the oxidation of substances by scavenging radicals, especially reactive oxygen species (ROS). Made up of one or two unpaired electrons, ROS are free radicals that are highly reactive and can attack other metabolites. By using electron paramagnetic resonance (EPR) spectroscopy, it is possible to measure paramagnetic substances such as free radicals. Therefore the dermal antioxidant activity can be determined by applying semi-stable radicals onto the skin and measuring the antioxidant-induced radical scavenging activity in the skin. In recent years, EPR has been
\end{abstract}


developed as a spectroscopic method for determining the antioxidant status in vivo. Several studies have shown that an additional uptake of dietary supplements, such as carotenoids or vitamin $\mathrm{C}$ in physiological concentrations, provide a protective effect against free radicals. Using the EPR technique it could be demonstrated that the radical production in stress situations, such as irradiation with infrared and visible light, was reduced with time. However, not only the oral uptake of antioxidants, but also the topical application of antioxidants, e.g., a hyperforin-rich cream, is very useful against the development of oxidative stress. Regular application of a hyperforin-rich cream reduced radical formation. The skin lipids, which are very important for the barrier function of the skin, were also stabilized.

Keywords: electron paramagnetic resonance (EPR) spectroscopy; free radicals; reactive oxygen species (ROS); antioxidants; carotenoids

\section{Introduction}

Reactive oxygen species (ROS) are essential for biological functions such as the respiratory chain, signal transfer and immune response. However, if the ROS exceed a certain level, negative effects can occur.

The influence of ROS on premature skin aging and tumor incidence is well-known [1]. The skin is confronted with ROS and other free radicals on a daily basis; whereby, the main noxa is solar radiation but also smoking, alcohol consumption and physical and psychological stress lead to the formation of ROS and free radicals in the skin-known as oxidative stress [2]. Oxidative stress promotes the damage of cell components by ROS such as hydrogen peroxide $\left(\mathrm{H}_{2} \mathrm{O}_{2}\right)$, hydroxyl radicals $(\mathrm{OH} \bullet)$ or superoxide radicals $\left(\mathrm{O}_{2} \bullet\right)$ [2-4]. Antioxidants as free radical scavengers can protect cells against the consequences of oxidative stress. They interrupt the radical chain reaction by giving up an electron to the ROS [5]. The antioxidants themselves immediately return to their antioxidant form and thus ensure an abrupt end to the dangerous radical chain reaction in the body [6]. If there are enough antioxidants available, the cells remain protected. An imbalance between the body's antioxidant defense system and the reactive metabolites induces several physiological processes within the body which can lead to e.g., cell aging, tissue damage or cancer [6-9]. If a strong interference between ROS and cells exists, programmed cell death (apoptosis) can be initiated [3,10,11]. Thereby the ROS can destroy several biomolecules in their function, such as lipids, proteins, enzymes or nucleic acid, promoting, inter alia, a peroxidation of the mitochondrial membrane lipids, leading to a mitochondrial permeability transition (MPT). MPT increases the permeability of the inner mitochondrial membrane, whereby larger molecules can affect the respiratory chain, thus the energy supply is interrupted $[12,13]$.

In general, one can distinguish between the enzymatic and non-enzymatic antioxidant defense systems in the body. Enzymatic antioxidants include, amongst others, the superoxide dismutase, glutathione peroxidase and catalase. Non-enzymatic antioxidants can be differentiated by endogenous (e.g., glutathion) and exogenous antioxidants. Exogenous antioxidants must be supplied with food, such as vitamin $\mathrm{C}$ and $\mathrm{E}$, carotenoids ( $\alpha$-carotene, $\beta$-carotene, lycopene, lutein), polyphenols (e.g., flavonoids) and trace elements (e.g., selenium, zinc) [14,15]. Today, the oral intake of antioxidants seems to be an effective source for protecting the body against oxidative stress [16-18]. 
One particular question is how the antioxidant status with special regard to the human skin can be enhanced to counteract the formation of ROS. A lot of products promise antioxidant protection, but do they in fact provide this protection? There are only few methods available to prove their effectiveness. Most of these are indirect methods, requiring blood or skin biopsies or they are performed in vitro with the uncertainty of whether they are representative for the in vivo situation. In this review, the in vivo electron paramagnetic resonance (EPR) spectroscopy will be presented as a non-invasive method to determine the radical scavenging capacity of the skin and the protection of stress, here exemplarily in form of irradiation in the visible/near infrared (VIS/NIR) spectral range because several studies suggest that beneficial effects of antioxidant products clearly extend beyond UV protection [19]. First measurements have already been performed using in vivo EPR spectroscopy [20-22] but only on single volunteers or small cohorts [23-25]. In this context, three studies will be presented and discussed where antioxidants were applied orally or topically in statistically evaluated clinical studies involving 20 or more subjects for providing a statistical significance of the results. The differences between the in vivo and ex vivo investigations are also discussed.

\section{Technical Aspects of the Performance of In Vivo EPR-Measurements}

Non-invasive EPR is based on the absorption of microwave radiation by paramagnetic substances in an externally applied static magnetic field [26-28]. Microwave absorption occurs when the applied microwave radiation corresponds to the energy difference between the spin states of an unpaired electron (Zeeman effect) [19,28,29]. Paramagnetic substances contain unpaired electrons, allowing free radicals and ROS in the skin to be detected [29]. Spin marker and spin traps in combination with L- and S-band EPR are often used for the analysis of radical production, $\mathrm{pH}$ measurements or oximetry in vivo/ex vivo [22,30-35]. The studies reviewed herein focused on the determination of the antioxidant potential in vivo. All the non-invasive in vivo measurements discussed in this review article were performed with an L band EPR spectrometer LBM MT 03 (Magnettech GmbH, Berlin, Germany) that is capable of measuring the whole arm of a volunteer in vivo [27].

Semi-stable radicals can be used to investigate the radical scavenging capacity of the skin [28]. For this technique, the spin marker TEMPO (2,2,6,6-tetramethylpiperidine-1-oxyl) is applied on the volar forearm and the decrease in the EPR signal is monitored over time [27]. The antioxidants react with the spin probe and reduce it to the corresponding EPR silent hydroxylamine. The antioxidant defense system of the skin depletes the radical molecules [36,37]. The decay of the EPR signal intensity can be expressed as a simple exponential function $I=I \exp (-\mathrm{k} t)$, where $\mathrm{k}$ is the rate constant. A high rate constant represents a high antioxidant capacity of the skin. A comparison between the baseline measurement, prior and subsequent to the topical application or oral intake of antioxidants, provides an insight into the radical scavenging capacity of the skin and how the intake of antioxidants have improved the rate constant $[27,32,35,38,39]$. To carry out oxidative stress measurements, the stable spin probe 3-(carboxy)-2,2,5,5-tetramethylpyrrolidin-1-oxyl (PCA) is applied onto the skin. This spin probe reacts with short-lived radicals produced by stress, such as irradiation. PCA does not directly react with antioxidants [40]. The difference between the measured values of the irradiated and non-irradiated probes reveals the stress-induced radical formation. With this technique, it is possible to determine the protective effect of applied antioxidants or sunscreen products against oxidative stress; 
applied antioxidants can react with the short-lived radicals whereby the PCA signal decreases less drastically. The topical application of a sunscreen could produce a secondary effect: The radical formation might be reduced due to physical filters which scatter the light before they can penetrate the Stratum corneum $(\mathrm{SC})$ to induce additional radicals [41,42].

\section{Discussion}

\subsection{Effect of Radical Formation in the Visible and Near Infrared Spectral Range}

Measurements of radiation-induced radical formation in the skin are representative for an interesting field regarding sun protection, photodynamic therapy, or radiation therapy [43]. It is well-known that solar radiation (UVA and UVB light) promotes the formation of free radicals in the skin [42]. However, considering the total amount of energy that reaches the earth, it was demonstrated that the UV light accounts for only 6.8\% (0.5\% UVB, 6.3\% UVA) and the visible light for only $38.9 \%$ of the radiation exposure. The infrared (IR) spectral range accounts for $54.3 \%$, and the NIR (as part of the IR spectral range) wavelength range for $30 \%$ of the radiation exposure [44,45]. In healthy skin, only a small amount of UVB irradiation penetrates into skin layers deeper than the epidermis. However, UVA has a higher penetration of the dermis, whereas NIR even penetrates into the subcutaneous tissue. Approximately 50\% of the NIR irradiation that reaches the skin is absorbed in the dermis [2] promoting premature skin aging [44-46]. It has been found that oxidative stress is induced not only in the UV, but also in the NIR spectral range [47]. This effect was observed by a depletion of dermal carotenoids after NIR irradiation [48]. This could not be explained by a direct radical formation, as the energy of the photons is too low. Several studies have confirmed that NIR irradiation leads to an upregulation of the matrix metalloproteinase 1 (MMP-1) expression in human skin promoting photoaging $[2,49]$. Therefore, NIR light can be used as a stress source to study the effect of topically applied or orally applied antioxidants concerning the improvement of the antioxidant status of the skin. Using in vivo EPR studies it was possible to analyze the biological response of supplements regarding their radical scavenging activity, whereby the radicals were induced by an external stress source, such as VIS/NIR irradiation. Studies with in situ stress induction were performed for the first time. The in situ measurements are a prerequisite to ensure reliable results allowing the response of the skin to be measured simultaneously.

Radiation as a stress source can be used to investigate the effect of topically applied and orally ingested antioxidants (Figure 1). In principle UV light could also be used to induce stress. Because of ethical reasons and the interesting protection effects beyond the UV wavelength range, the study was performed using VIS/NIR light. 


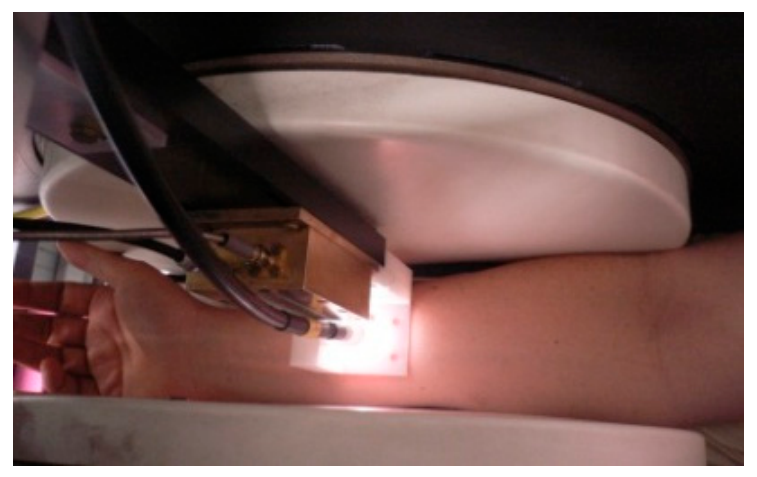

Figure 1. In vivo EPR system with in situ fiber-guided radiation of a solar simulator.

\subsection{Effect of Orally Ingested Antioxidants}

Vitamin $\mathrm{C}$ is a potential radical scavenger and one of the best known antioxidants in mammal organisms [50]. It is found naturally in many fruits and vegetables, such as citrus fruits and peppers [51] but also ascorbic acid (E 300) and sodium ascorbate (E 301) as food additives (preservatives) should be mentioned as vitamin $\mathrm{C}$ sources. However, vitamin $\mathrm{C}$ is often used as a component of dietary supplements. It is also known that vitamin $\mathrm{C}$ accumulates in the skin and can reduce photo damage after systemic or topical application [52]. Therefore, it can be used as a positive control in studies on the reduction of radical formation in the skin. In one of the recent studies, the radical scavenging capacity of the human skin on the inner right forearm was examined on the baseline and four weeks after supplementation in response to supplementation with moderate vitamin $\mathrm{C}$ doses [53-55]. A total number of 33 healthy volunteers were given either supplements with vitamin $\mathrm{C}$, a chokeberry peel extract (Aronia melanocarpa), or a placebo for four weeks [54]. The amount of supplementation corresponded to $125 \mathrm{mg}$ of calcium ascorbate per day or $800 \mathrm{mg}$ of Aronia melanocarpa combined with $40 \mathrm{mg}$ of calcium ascorbate. The administered vitamin $\mathrm{C}$ doses that were ingested by the volunteers in addition to their usual diet were consistent with the standards of the US Food and Nutrition Board and the German Nutrition Society [53]. An oral supplementation for four weeks with vitamin $\mathrm{C}$ and Aronia melanocarpa significantly increased the radical scavenging capacity of the skin by $22 \%$ and $23 \%$, respectively (Figure 2 ).

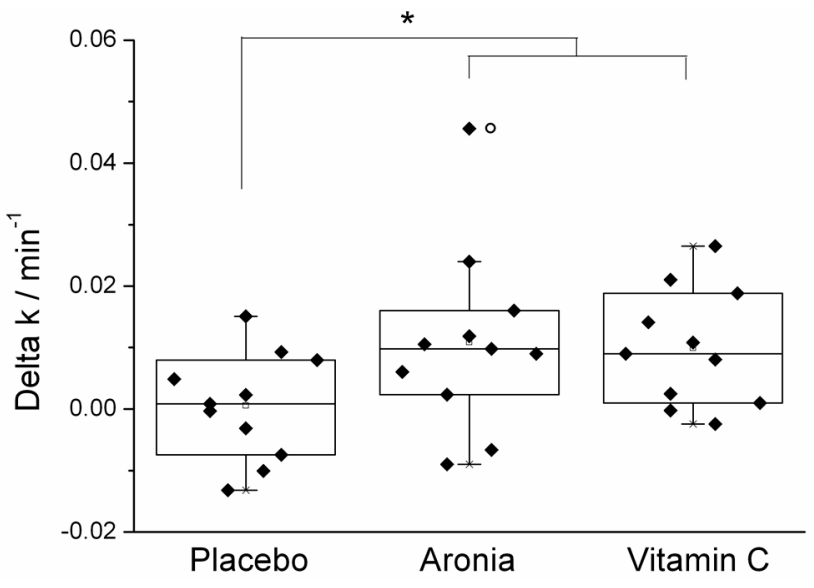

Figure 2. Rate constant after four week intake of verum and the placebo normalized to the initial values [55], $* p<0.05$. 
Thereby, the decrease of the signal intensity of the spin probe TEMPO was measured over time. The placebo group showed no alteration in their rate constant during the entire study [54]. In addition to these results, different vitamin $\mathrm{C}$ doses (dose 1: $100 \mathrm{mg}$ /day; dose 2: $180 \mathrm{mg} /$ day) were compared for their efficiency [53]. In this context, the radical scavenging properties of the antioxidant vitamin $\mathrm{C}$ were examined using EPR technology after two and four weeks, respectively. After four weeks, a significant increase of $22 \%$ (for dose 1) and $37 \%$ (for dose 2) of the rate constant was clearly visible. The placebo control group showed no change in their antioxidant activity, indicating a clear improvement of the radical scavenging activity of the skin in the group with the additional uptake of vitamin C. Further to the in vivo measurements, the radical scavenging capacity of the administered substances, described as radical protection factor (RPF), were investigated by in vitro EPR [55]. The RPF can be determined by the reaction of the test substance with the radical probe 2,2-diphenyl-1-picrylhydrazyl (DPPH) and is defined as $3600 \times 10^{14}$ (neutralized radicals)/mg (test substance) [38]. This procedure is a good measure for evaluating the antioxidant potency of a preparation. Aronia exhibited an RPF value of 3600 . The lower dose of vitamin $\mathrm{C}$ was adapted to the RPF factor of Aronia. The second vitamin $\mathrm{C}$ dose showed a 1.8 times higher RPF [55]. A dose-dependence of the rate constant was clearly demonstrated, and, after two weeks, the rate constant reached its maximum [53]. The EPR technology gives an insight into the reaction towards radicals. An increased intake of exogenous antioxidants improves the antioxidant activity of the skin.

Shindo et al. (1993) show that the vitamin C concentration in the epidermis is six times higher than in the dermis [56]. The increased vitamin $\mathrm{C}$ concentration in the outermost skin layer can be explained by the peroxidative environment to which the skin is exposed [57]. In recent studies, the EPR technology was successfully used for the in vivo determination of the radical scavenging capacity of the skin after oral supplementation with antioxidants. It has been demonstrated that the administration of a water soluble extract (Aronia melanocarpa and vitamin C) can improve the antioxidant status of the skin. Both pure synthetic vitamin $\mathrm{C}$ and a natural mixed preparation containing mainly flavonoids and polyphenols improve the free radical scavenging properties of the skin in the same way as if applied with the same RPF. These results illustrated that even polyphenols, which are supposed to accumulate in the skin after oral administration, exhibit an antioxidant effect in the skin. It is worth mentioning that vitamin $\mathrm{C}$ and the natural product reduce the spin probe TEMPO also in vitro. No significant increase in the carotenoid concentration was detected subsequent to supplementation with pure vitamin $\mathrm{C}$ and the mixed preparations after four weeks using resonance Raman spectroscopy. Long-term effects were not assessed as the period of study was too short [53-55].

While the first study showed that EPR is able to show the effect of water-soluble antioxidants, the behavior of applied lipophilic antioxidants, which do not react with the spin probe in vitro, was addressed in a second study.

Cutaneous carotenoids are lipophilic antioxidants and serve as a superior marker for the antioxidant capacity of the body [58,59]. Carotenoids such as most of the known antioxidants have to be taken up with food, such as fruits and vegetables, because they cannot be produced by the body itself $[60,61]$. To investigate the effect of lipophilic antioxidants on the spin probe TEMPO, a double-blinded placebo-controlled study with 24 healthy volunteers was carried out, all of which were given a supplementary lipophilic curly kale extract (Lutex Skin) for 8 weeks [38]. In addition to resonance Raman spectroscopy, which is usually applied for detecting the carotenoid concentration in the skin (Figure 3a), EPR spectroscopy was used for measuring the antioxidant status in the skin (Figure 3b). 
(a)

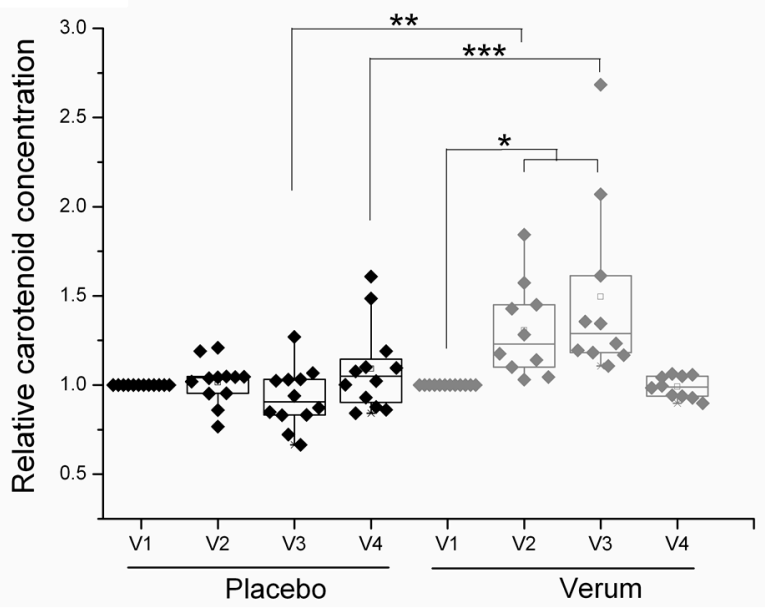

(b)

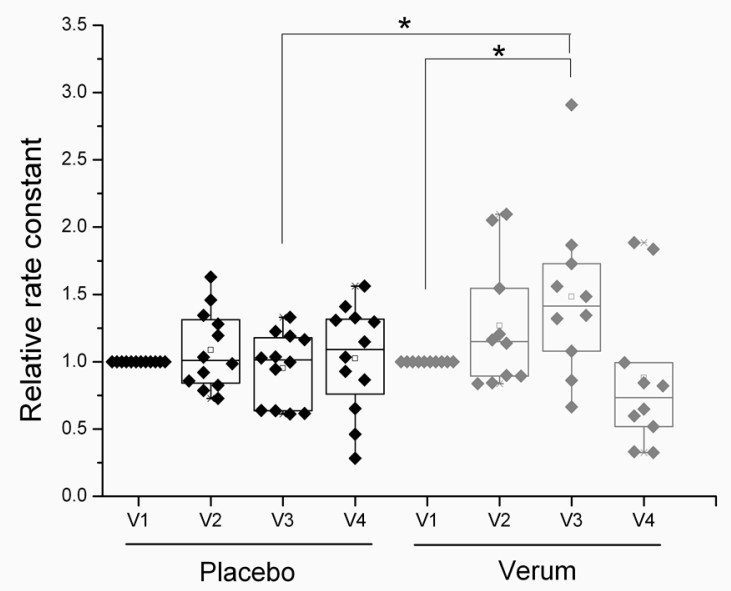

Figure 3. Data taken from visits 1 to 4 (V1, before intake; V2, four weeks after intake; V3, eight weeks after intake; V4, several weeks after discontinuing the intake; (a) relative carotenoid concentration; (b) relative rate constant [38], * $p<0.05, * * p<0.01$, *** $p<0.001$.

The rate constant in the verum group was increased, and after 10 min of irradiation with light in the VIS/NIR spectral range, an improved protection against VIS/NIR-induced radicals was detected by EPR. Eight weeks of supplementation with natural carotenoids reduced the radical formation by the VIS/NIR irradiation to 34\% (Figure 3b). The EPR results were consistent with the results of the resonance Raman spectroscopy [38]. This reduction occurred although the applied EPR marker did not react with the applied antioxidants, which clearly suggests that the whole antioxidant level was enhanced. Therefore, the carotenoids could serve as a marker substance of the whole antioxidant potential. The antioxidant-rich extract had no significant impact on the skin lipids [38].

A comparison of the results of the recent studies on hydrophilic and lipophilic antioxidants [38,53-55] clearly showed that after an oral intake of water-soluble antioxidants, the antioxidant potential of the skin increased within only four weeks, whereas lipophilic antioxidants needed eight weeks to become fully effective. An enrichment of the skin with carotenoids via an oral supplementation for eight weeks increased the free radical scavenging properties of the skin fairly well [38].

\subsection{Effect of Topical Application}

The topical application of drugs provides an effective method for treating skin diseases. Free radicals play an important role in, for example, atopic dermatitis and Lichen planus [62,63]. The topical application of antioxidants is therefore a promising tool to reduce the radical overload in the skin. Moreover, daily skin care is essential to maintain the skin barrier or to support its regeneration [64]. Hyperforin provides anti-inflammatory, antitumor and antibacterial properties and is a major constituent of St. John's Wort (Hypericum perforatum) $[65,66]$. It also reduces the free radical formation in the skin after exposure to UV and IR radiation [42]. In our studies, the protection effect of topically applied antioxidants was examined compared to increased radical formation in skin induced by VIS/NIR irradiation via the EPR marker PCA [40,64]. 
A hyperforin-rich cream was analyzed for its radical protection in the skin after VIS/NIR irradiation and compared to untreated skin and placebo samples [40,64]. Eleven volunteers with healthy skin were examined after a single, and further four-week cream application. A positive short-term and a long-term effect on the radical protection and skin lipids were demonstrated [40]. One hour after a single cream application, the radical formation after irradiation with VIS/NIR was completely inhibited by both verum and a placebo cream [40]. It is therefore reasonable to assume that the excellent immediate protective effect of both creams is due to their scattering properties rather than to the addition of hyperforin. Moreover, after one hour, the skin surface was still covered with a cream layer which could have provided protection against the radical formation induced by VIS/NIR irradiation (Figure 4a) [19]. The EPR results after long-term usage differed significantly from those obtained after a single cream application. A four-week application of the hyperforin-rich cream significantly reduced the radical formation in skin; for the placebo, a $45 \%$ radical reduction was detectable, for verum, the radical formation was reduced by up to $78 \%$ (Figure $4 b$ ).

(a)

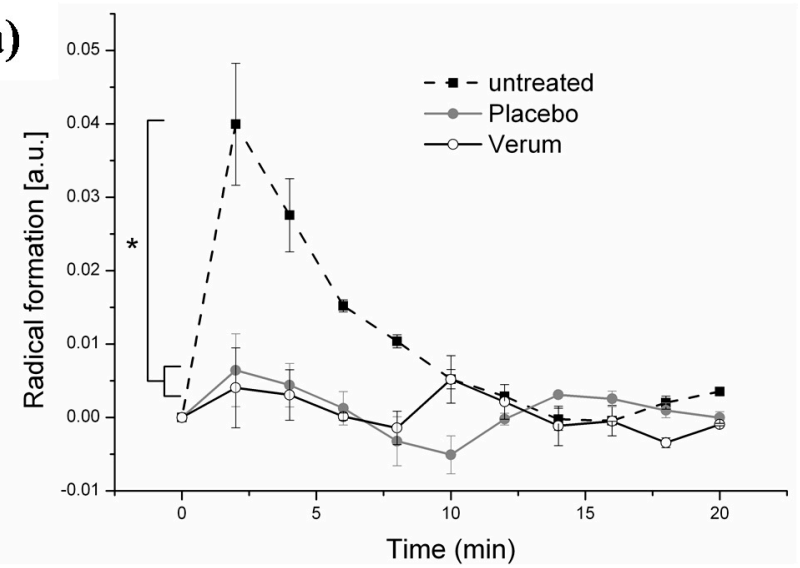

(b)

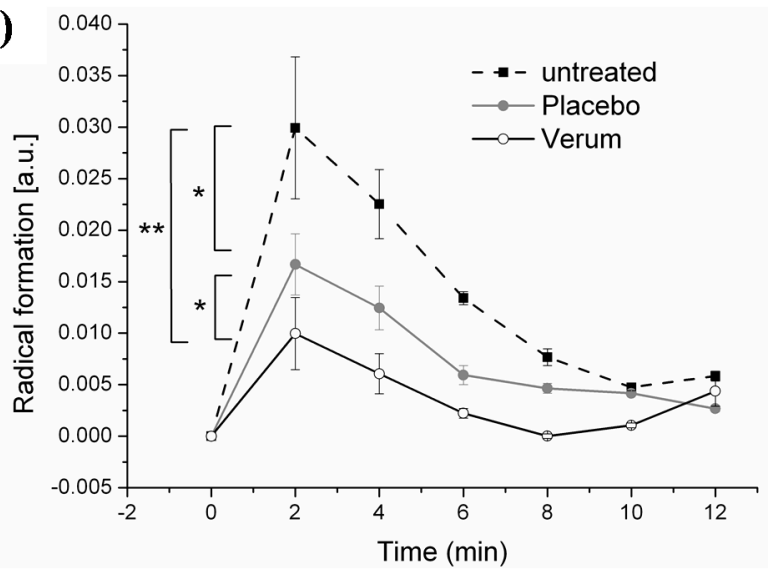

Figure 4. (a) Radical formation after single application $(* p \leq 0.05)$ and (b) after four weeks of twice daily application of the creams $(* p \leq 0.05, * * p \leq 0.01)$ [40].

The hyperforin-rich cream has an RPF of $425 \times 10^{14}$ radicals $/ \mathrm{mg}$, in contrast to the placebo cream with an RPF of $39 \times 10^{14}$ radicals/mg [40,42]. A high radical scavenging effect was detected with a linear function to the hyperforin concentration $(0.5 \%-5 \%)$ [19]. The high antioxidant potential of the skin treated with the verum cream is most likely explainable by the ingredient hyperforin, which has already been proved in a previous study analyzing the antioxidant properties by an UV erythema test in vivo and ex vivo and subsequent EPR measurements [42]. The investigations were performed 5 to $14 \mathrm{~h}$ after the last application; a full day protection was observed. In contrast, a single application did not provide $24 \mathrm{~h}$ protection; after $5 \mathrm{~h}$ the effect had vanished.

The kinetics of the radical production-strong increase at the beginning and decrease after $2 \mathrm{~min}$ leads to the assumption that the body responds to the induced stress. Of course, such effects could be beneficial if the induced stress is moderate.

In addition to the stabilization of the antioxidant network of the skin, concentration of skin lipids such as cholesterol and ceramides, which are important for an intact skin barrier, increased (measured by high-performance thin-layer chromatography (HPTLC). Hyperforin and, to a lesser extent, also the antioxidants in the base cream formulation appeared to interact with the antioxidant 
network of the Stratum corneum (SC) and led to a stabilization of the skin barrier function [40]. This study supports former studies which had shown that antioxidants which were applied topically are able to reduce the radical formation during VIS/NIR irradiation $[19,42]$.

Schempp et al. studied the effect of an antioxidant-containing cream, a base and barrier protection cream on untreated skin in order to analyze the hydration of the SC and the blood flow by a chemically induced contact dermatitis [67]. The best effect was achieved with the antioxidant containing cream, whereas the untreated skin showed the worst results. Other studies with creams containing antioxidants also showed positive skin effects with respect to contact allergies and contact dermatitis [67-70].

In our study, the radical formation was reduced by almost $80 \%$ by applying a hyperforin-rich cream. Furthermore, a full-day time effect was validated after an application time of four weeks. In general, it was shown that a regular application of antioxidant-containing creams is necessary to obtain a fully effective radical protection and to stimulate the antioxidant defense system to counteract any oxidative stress.

\subsection{Comparison of In Vivo and Ex Vivo Investigations}

Dermal applications of drugs on the skin are of increasing importance in human medicine to counteract certain skin diseases, e.g., acne or psoriasis. In order to find suitable substances and to check their effectiveness, it is necessary to analyze the behavior of drugs ex vivo and in vivo [71]. Often, in vivo experiments display some problems; the reproducibility is often insufficient, most test products are denied official approval and the experiments are difficult to handle, especially when exposure to radiation is involved. Up to now, most irradiation experiments have been performed ex vivo, only a few studies have been conducted on volunteers in vivo [37,72,73]. Taking into consideration the risk of erythema development, the formation of free radicals in the radiation-exposed skin can only be investigated for a limited time, in vivo. Thus ex vivo experiments are very helpful in understanding the kinetics of radical formation during irradiation, because the samples do not have to be removed for erythema development [64]. In dermatology, pig ear skin is commonly used as a human skin model [74]. With the EPR technology, it is possible to measure the radical formation in skin in vivo as well as ex vivo [38,64]. In our study, the radical protection of skin in the visible and infrared spectral range by a hyperforin-rich cream was studied in vivo vs. ex vivo, non-treated skin was measured as control [64]. The radical scavenging capacity of the verum cream was determined for the RPF by EPR measurements and showed a linear function to the hyperforin concentration [19].

A comparison between in vivo and ex vivo was carried out after a single cream application for the pig ear and after four weeks for in vivo (human skin). It was demonstrated that both creams, verum and placebo, protected the skin from VIS/NIR-induced radicals, but the absolute values and kinetics for in vivo vs. ex vivo investigations, with regard to the radical formation, were not comparable (Figure 5).

The effect of the placebo cream could be explained by the optical properties of the base cream. In addition to the included antioxidants, high scattering properties could be the cause of reduced radical formation for placebo. This effect was successfully demonstrated in ex vivo studies for sunscreens where physical filters were ingredients of the cream [19]. During in vivo studies, the radical production increased quickly but decreased with time after VIS/NIR irradiation. In the ex vivo studies, it remained stable over the investigated time period. This effect is explainable by the loss of oxygen supply ex vivo [41,64]. 
The stronger decrease in time in vivo could be also explained by a possible up-regulation of the endogenous antioxidant network in the skin, whereby the induced radicals are neutralized [10]. Furthermore, reduced enzymatic and cell activity after excision intensifies the differences between in vivo and ex vivo [64]. Regensburger et al. (2012) showed that ROS formed by vitamins could behave in different ways ex vivo and in vivo [75]. In general, ex vivo show a lower absolute radical production compared to in vivo, but the proportions were quite similar to each other [64]. These results were confirmed by further studies [41].

Ex vivo and in vivo studies occupy a very important position in the research of the development of ROS in the skin and provide a first hypothesis of the effect of external stress induction and how topically applied antioxidants can influence the antioxidant network of the skin. One should keep in mind that ex vivo skin does not behave in a way that is directly comparable to in vivo skin, but it can indicate a tendency.

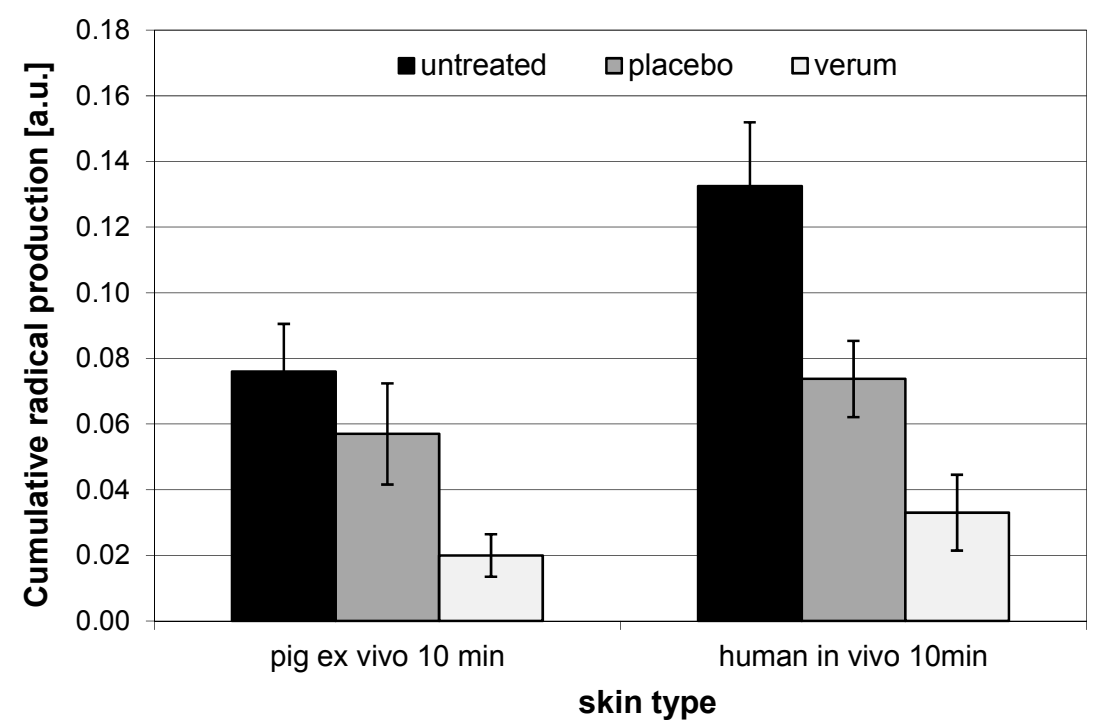

Figure 5. Cumulative radical production in excised pig ear and human in vivo investigations. Modified and reproduced with permission from [64], published by John Wiley and Sons, 2013.

\section{Conclusion}

The EPR technique is now a very important and well-established in vivo and ex vivo method in dermatology for measuring the development of free radicals and the antioxidant potential of the skin under in situ conditions.

Several studies have demonstrated that systemic administration but also topical application of antioxidants can enhance the radical scavenging properties of the skin. Ex vivo EPR measurements give a very good primary indication of the influence of topically applied antioxidants for the antioxidant network of the skin and therefore display a good alternative to in vivo investigations. Long-term effects can only be studied in in vivo experiments. A combination of various non-invasive methods (e.g., EPR, resonance Raman spectroscopy) in vivo and ex vivo provides an effective approach to determine the antioxidant potential of the skin. The EPR technology enriches the field of skin antioxidant evaluation. Orally applied water soluble antioxidants (such as vitamin $\mathrm{C}$ ) increase the 
antioxidative status of the skin very quickly, lipophilic antioxidants (such as carotenoids) require more time to show their protecting effect against ROS. Topically applied antioxidants showed the highest protection efficiency but only on the applied areas. These results indicate that a diet rich in antioxidants enhances the skin protection against free radicals and therefore provides protection against premature skin aging and radical associated skin diseases.

\section{Acknowledgments}

The authors acknowledge the foundation "Skin physiology" of the Donor Association for German Science and Humanities for financial support. A part of the work was supported by the Federal Land of Berlin within the Programm to Promote Research, Innovation and Technologies ProFIT (grant No. 10142343 and 10149524) for granting subsidies from the innovation promotion fund with cofinancing by the European Fund for Regional Development ERDF.

\section{Author Contributions}

Martina Claudia Meinke, Jürgen Lademann, Henning Vollert, Norbert Groth designed research; Henning Vollert, Norbert Groth, Anke Kleemann prepared samples; Anna-Christina Lauer, Sophia Arndt, Annette Friedrich performed EPR research; Kathrin Tscherch and Sascha Rohn performed the lipid analysis; Ingo Gersonde, Martina Claudia Meinke, Stefan F. Haag and Maxim E. Darvin analyzed data; Silke Barbara Lohan and Martina Claudia Meinke have written the paper.

\section{Conflicts of Interest}

The authors declare no conflict of interest.

\section{References}

1. Chen, L.; Hu, J.Y.; Wang, S.Q. The role of antioxidants in photoprotection: A critical review. J. Am. Acad. Dermatol. 2012, 67, 1013-1024.

2. Zastrow, L.; Groth, N.; Klein, F.; Kockott, D.; Lademann, J.; Renneberg, R.; Ferrero, L. The missing link-light-induced $(280-1600 \mathrm{~nm})$ free radical formation in human skin. Skin Pharmacol. Physiol. 2009, 22, 31-44.

3. Gutteridge, J.M.; Halliwell, B. Free radicals and antioxidants in the year 2000: A historical look to the future. Ann. N.Y. Acad. Sci. 2000, 899, 136-147.

4. Lademann, B.; Gerber, D.M.; Olbertz, M.E.; Darvin, L.; Stauf, K.; Ueberholz, V.; Heinrich, J.; Lademann, V. Non-invasive spectroscopic determination of the antioxidative status of gravidae and neonates. Skin Pharmacol. Physiol. 2015, 28, 189-195.

5. Wolfle, U.; Seelinger, G.; Bauer, G.; Meinke, M.C.; Lademann, J.; Schempp, C.M. Reactive molecule species and antioxidative mechanisms in normal skin and skin aging. Skin Pharmacol. Physiol. 2014, 27, 316-332.

6. Sies, H. Oxidative stress: Oxidants and antioxidants. Exp. Physiol. 1997, 82, 291-295.

7. Briganti, S.; Picardo, M. Antioxidant activity, lipid peroxidation and skin diseases. What's new. J. Eur. Acad. Dermatol. Venereol. 2003, 17, 663-669. 
8. Harman, D. Aging: A theory based on free radical and radiation chemistry. J. Gerontol. 1956, 11, 298-300.

9. Sander, C.S.; Chang, H.; Hamm, F.; Elsner, P.; Thiele, J.J. Role of oxidative stress and the antioxidant network in cutaneous carcinogenesis. Int. J. Dermatol. 2004, 43, 326-335.

10. Thiele, J.J.; Schroeter, C.; Hsieh, S.N.; Podda, M.; Packer, L. The antioxidant network of the stratum corneum. Curr. Probl. Dermatol. 2001, 29, 26-42.

11. Lademann, J.; Kocher, W.; Yu, R.; Meinke, M.C.; Na Lee, B.; Jung, S.; Sterry, W.; Darvin, M.E. Cutaneous carotenoids: The mirror of lifestyle? Skin Pharmacol. Physiol. 2014, 27, 201-207.

12. Zamzami, N.; Kroemer, G. The mitochondrion in apoptosis: How Pandora's box opens. Nat. Rev. Mol. Cell Biol. 2001, 2, 67-71.

13. Cai, J.; Jones, D.P. Mitochondrial redox signaling during apoptosis. J. Bioenerg. Biomembr. 1999, $31,327-334$.

14. Lademann, J.; Patzelt, A.; Schanzer, S.; Richter, H.; Meinke, M.C.; Sterry, W.; Zastrow, L.; Doucet, O.; Vergou, T.; Darvin, M.E. Uptake of antioxidants by natural nutrition and supplementation: Pros and cons from the dermatological point of view. Skin Pharmacol. Physiol. 2011, 24, 269-273.

15. Shindo, Y.; Witt, E.; Han, D.; Epstein, W.; Packer, L. Enzymic and non-enzymic antioxidants in epidermis and dermis of human skin. J. Invest. Dermatol. 1994, 102, 122-124.

16. Uddin, S.; Ahmad, S. Antioxidants protection against cancer and other human diseases. Compr. Ther. 1995, 21, 41-45.

17. Robert, C.; Bonnet, M.; Marques, S.; Numa, M.; Doucet, O. Low to moderate doses of infrared A irradiation impair extracellular matrix homeostasis of the skin and contribute to skin photodamage. Skin Pharmacol. Physiol. 2015, 28, 196-204.

18. Park, G.; Kim, H.G.; Hong, S.P.; Kim, S.Y.; Oh, M.S. Walnuts (seeds of Juglandis sinensis L.) protect human epidermal keratinocytes against UVB-induced mitochondria-mediated apoptosis through upregulation of ROS elimination pathways. Skin Pharmacol. Physiol. 2014, 27, 132-140.

19. Meinke, M.C.; Haag, S.F.; Schanzer, S.; Groth, N.; Gersonde, I.; Lademann, J. Radical protection by sunscreens in the infrared spectral range. Photochem. Photobiol. 2011, 87, 452-456.

20. He, G.; Samouilov, A.; Kuppusamy, P.; Zweier, J.L. In vivo imaging of free radicals: Applications from mouse to man. Mol. Cell. Biochem. 2002, 234, 359-367.

21. Gallez, B.; Swartz, H.M. In vivo EPR: When, how and why? NMR Biomed. 2004, 17, 223-225.

22. Fujii, H.; Berliner, L.J. Detection of bioradicals by in vivo L-band electron spin resonance spectrometry. NMR Biomed. 2004, 17, 311-318.

23. Zastrow, T.H.L.; Berliner, L.J.; Ferrero, L.; Groth, N. In-vivo measurements of free radicals in human skin. IFSCC Mag. 2003, 6, 295-301.

24. He, G.; Samouilov, A.; Kuppusamy, P.; Zweier, J.L. In vivo EPR imaging of the distribution and metabolism of nitroxide radicals in human skin. J. Magn. Reson. 2001, 148, 155-164.

25. Herrling, T.; Jung, K.; Fuchs, J. Measurements of UV-generated free radicals/reactive oxygen species (ROS) in skin. Spectrochim. Acta A Mol. Biomol. Spectrosc. 2006, 63, 840-845. 
26. Haag, S.F.; Taskoparan, B.; Bittl, R.; Teutloff, C.; Wenzel, R.; Fahr, A.; Chen, M.; Lademann, J.; Schafer-Korting, M.; Meinke, M.C. Stabilization of reactive nitroxides using invasomes to allow prolonged electron paramagnetic resonance measurements. Skin Pharmacol. Physiol. 2011, 24, 312-321.

27. Haag, S.F.; Taskoparan, B.; Darvin, M.E.; Groth, N.; Lademann, J.; Sterry, W.; Meinke, M.C. Determination of the antioxidative capacity of the skin in vivo using resonance Raman and electron paramagnetic resonance spectroscopy. Exp. Dermatol. 2011, 20, 483-487.

28. Vogt, O.; Lademann, J.; Rancan, F.; Meinke, M.C.; Schanzer, S.; Stockfleth, E.; Sterry, W.; Lange-Asschenfeldt, B. Photoprotective properties of the fluorescent europium complex in UV-irradiated skin. Skin Pharmacol. Physiol. 2013, 26, 76-84.

29. Weil. J.A.; Bolton, J.R.; Wertz, J.E. Electron Paramagnetic Resonance; Wiley-Interscience: Hoboken, NY, USA, 1994.

30. Swartz, H.M.; Khan, N.; Buckey, J.; Comi, R.; Gould, L.; Grinberg, O.; Hartford, A.; Hopf, H.; Hou, H.; Hug, E.; et al. Clinical applications of EPR: Overview and perspectives. NMR Biomed. 2004, 17, 335-351.

31. Berliner, L.J.; Fujii, H. In vivo spin trapping of nitric oxide. Antioxid. Redox Signal. 2004, 6, 649-656.

32. Gallez, B.; Bacic, G.; Goda, F.; Jiang, J.; O’Hara, J.A.; Dunn, J.F.; Swartz, H.M. Use of nitroxides for assessing perfusion, oxygenation, and viability of tissues: In vivo EPR and MRI studies. Magn. Reson. Med. 1996, 36, 97-106.

33. Gallez, B.; Mader, K.; Swartz, H.M. Noninvasive measurement of the $\mathrm{pH}$ inside the gut by using pH-sensitive nitroxides. An in vivo EPR study. Magn. Reson. Med. 1996, 36, 694-697.

34. Ibragimova, M.I.; Petukhov, V.Y.; Zheglov, E.P.; Khan, N.; Hou, H.; Swartz, H.M.; Konjukhov, G.V.; Nizamov, R.N. Quinoid radio-toxin (QRT) induced metabolic changes in mice: An ex vivo and in vivo EPR investigation. Nitric Oxide 2008, 18, 216-222.

35. Fuchs, J.; Groth, N.; Herrling, T. In vivo measurement of oxidative stress status in human skin. Methods Enzymol. 2002, 352, 333-339.

36. Fuchs, J.; Freisleben, H.J.; Podda, M.; Zimmer, G.; Milbradt, R.; Packer, L. Nitroxide radical biostability in skin. Free Radic. Biol. Med. 1993, 15, 415-423.

37. Herrling, T.; Fuchs, J.; Rehberg, J. UV-induced free radicals in the skin detected by ESR spectroscopy and imaging using nitroxides. Free Radic. Biol. Med. 2003, 35, 59-67.

38. Meinke, M.C.; Friedrich, A.; Tscherch, K.; Haag, S.F.; Darvin, M.E.; Vollert, H.; Groth, N.; Lademann, J.; Rohn, S. Influence of dietary carotenoids on radical scavenging capacity of the skin and skin lipids. Eur. J. Pharm. Biopharm. 2013, 84, 365-373.

39. Herrling, T.; Zastrow, L.; Groth, N. Detection and Influencing of the Antioxidative Potential (AOP) of Human Skin. SÖFW 1996, 122, 472-476.

40. Haag, S.F.; Tscherch, K.; Arndt, S.; Kleemann, A.; Gersonde, I.; Lademann, J.; Rohn, S.; Meinke, M.C. Enhancement of skin radical scavenging activity and stratum corneum lipids after the application of a hyperforin-rich cream. Eur. J. Pharm. Biopharm. 2014, 86, 227-233. 
41. Meinke, M.C.; Muller, R.; Bechtel, A.; Haag, S.F.; Darvin, M.E.; Lohan, S.B.; Ismaeel, F.; Lademann, J. Evaluation of carotenoids and reactive oxygen species in human skin after UV irradiation: A critical comparison between in vivo and ex vivo investigations. Exp. Dermatol. 2015, 24, 194-197.

42. Meinke, M.C.; Schanzer, S.; Haag, S.F.; Casetti, F.; Muller, M.L.; Wolfle, U.; Kleemann, A.; Lademann, J.; Schempp, C.M. In vivo photoprotective and anti-inflammatory effect of hyperforinis associated with high antioxidant activity in vitro and ex vivo. Eur. J. Pharm. Biopharm. 2012, 81, 346-350.

43. Terman, M.; Terman, J.S. Light therapy for seasonal and nonseasonal depression: Efficacy, protocol, safety, and side effects. CNS Spectr. 2005, 10, 647-663.

44. Kochevar, I.E.; Pathak, M.A.; Parrish, J.A. Photophysics, photochemistry, and photobiology. In Fitzpatrick's Dermatology in General Medicine; Freedberg, I.M., Eisen, A.Z., Wolff, K., Eds.; McGraw-Hill: New York, NY, USA, 1999.

45. Schieke, S.M.; Schroeder, P.; Krutmann, J. Cutaneous effects of infrared radiation: From clinical observations to molecular response mechanisms. Photodermatol. Photoimmunol. Photomed. 2003, 19, 228-234.

46. Kligman, L.H. Intensification of ultraviolet-induced dermal damage by infrared radiation. Arch. Dermatol. Res. 1982, 272, 229-238.

47. Akhalaya, M.Y.; Maksimov, G.V.; Rubin, A.B.; Lademann, J.; Darvin, M.E. Molecular action mechanisms of solar infrared radiation and heat on human skin. Ageing Res. Rev. 2014, 16, 1-11.

48. Darvin, M.E.; Patzelt, A.; Meinke, M.C.; Sterry, W.; Lademann, J. Influence of two different IR radiators on the antioxidative potential of the human skin. Laser Phys. Lett. 2009, 6, 229-234.

49. Schroeder, P.; Lademann, J.; Darvin, M.E.; Stege, H.; Marks, C.; Bruhnke, S.; Krutmann, J. Infrared radiation-induced matrix metalloproteinase in human skin: Implications for protection. J. Invest. Dermatol. 2008, 128, 2491-2497.

50. Buettner, G.R.; Jurkiewicz, B.A. Catalytic metals, ascorbate and free radicals: Combinations to avoid. Radiat. Res. 1996, 145, 532-541.

51. Furst, A. Can nutrition affect chemical toxicity? Int. J. Toxicol. 2002, 21, 419-424.

52. Dayan, S.H.; Arkins, J.P.; Sharma, V.; Paterson, E.; Barnes, D. A phase 2, double-blind, randomized, placebo-controlled trial of a novel nutritional supplement product to promote healthy skin. J. Drugs Dermatol. 2011, 10, 1106-1114.

53. Lauer, A.C.; Groth, N.; Haag, S.F.; Darvin, M.E.; Lademann, J.; Meinke, M.C. Dose-dependent vitamin $\mathrm{C}$ uptake and radical scavenging activity in human skin measured with in vivo electron paramagnetic resonance spectroscopy. Skin Pharmacol. Physiol. 2013, 26, 147-154.

54. Lauer, A.C.; Groth, N.; Haag, S.F.; Darvin, M.E.; Lademann, J.; Meinke, M.C. Radical scavenging capacity in human skin before and after vitamin $\mathrm{C}$ uptake: An in vivo feasibility study using electron paramagnetic resonance spectroscopy. J. Invest. Dermatol. 2013, 133, 1102-1104.

55. Meinke, M.C.; Lauer, A.-C.; Haag, S.F.; Darvin, M.E.; Groth, N.; Lademann, J. Cutaneous radical scavenging effects of orally administered antioxidants measured by electron paramagnetic resonance spectroscopy. E-SPEN J. 2012, 7, 160-166. 
56. Shindo, Y.; Witt, E.; Packer, L. Antioxidant defense mechanisms in murine epidermis and dermis and their responses to ultraviolet light. J. Invest. Dermatol. 1993, 100, 260-265.

57. Gaziano, J.M.; Glynn, R.J.; Christen, W.G.; Kurth, T.; Belanger, C.; MacFadyen, J.; Bubes, V.; Manson, J.E.; Sesso, H.D.; Buring, J.E. Vitamins E and C in the prevention of prostate and total cancer in men: The Physicians' Health Study II randomized controlled trial. JAMA 2009, 301, 52-62.

58. Meinke, M.C.; Darvin, M.E.; Vollert, H.; Lademann, J. Bioavailability of natural carotenoids in human skin compared to blood. Eur. J. Pharm. Biopharm. 2010, 76, 269-274.

59. Darvin, M.E.; Fluhr, J.W.; Schanzer, S.; Richter, H.; Patzelt, A.; Meinke, M.C.; Zastrow, L.; Golz, K.; Doucet, O.; Sterry, W.; et al. Dermal carotenoid level and kinetics after topical and systemic administration of antioxidants: Enrichment strategies in a controlled in vivo study. J. Dermatol. Sci. 2011, 64, 53-58.

60. Lademann, J.; Schanzer, S.; Meinke, M.; Sterry, W.; Darvin, M.E. Interaction between carotenoids and free radicals in human skin. Skin Pharmacol. Physiol. 2011, 24, 238-244.

61. De Spirt, S.; Sies, H.; Tronnier, H.; Heinrich, U. An encapsulated fruit and vegetable juice concentrate increases skin microcirculation in healthy women. Skin Pharmacol. Physiol. 2012, 25, 2-8.

62. Sapuntsova, S.G.; Lebed'ko, O.A.; Shchetkina, M.V.; Fleyshman, M.Y.; Kozulin, E.A.; Timoshin, S.S. Status of free-radical oxidation and proliferation processes in patients with atopic dermatitis and lichen planus. Bull. Exp. Biol. Med. 2011, 150, 690-692.

63. Aly, D.G.; Shahin, R.S. Oxidative stress in lichen planus. Acta Dermatoven APA 2010, 19, 3-11.

64. Arndt, S.; Haag, S.F.; Kleemann, A.; Lademann, J.; Meinke, M.C. Radical protection in the visible and infrared by a hyperforin-rich cream-In vivo vs. ex vivo methods. Exp. Dermatol. 2013, 22, 354-357.

65. Schempp, C.M.; Windeck, T.; Hezel, S.; Simon, J.C. Topical treatment of atopic dermatitis with St. John's wort cream-A randomized, placebo controlled, double blind half-side comparison. Phytomedicine 2003, 10, 31-37.

66. Schempp, C.M.; Pelz, K.; Wittmer, A.; Schopf, E.; Simon, J.C. Antibacterial activity of hyperforin from St John's wort, against multiresistant Staphylococcus aureus and gram-positive bacteria. Lancet 1999, 353, 2129-2130.

67. Schempp, C.M.; Meinke, M.C.; Lademann, J.; Ferrari, Y.; Brecht, T.; Gehring, W. Topical antioxidants protect the skin from chemical-induced irritation in the repetitive washing test: A placebo-controlled, double-blind study. Contact Dermat. 2012, 67, 234-237.

68. Esser, P.R.; Wolfle, U.; Durr, C.; von Loewenich, F.D.; Schempp, C.M.; Freudenberg, M.A.; Jakob, T.; Martin, S.F. Contact sensitizers induce skin inflammation via ROS production and hyaluronic acid degradation. PLoS One 2012, 7, doi:10.1371/journal.pone.0041340.

69. Senaldi, G.; Pointaire, P.; Piguet, P.F.; Grau, G.E. Protective effect of $N$-acetylcysteine in hapten-induced irritant and contact hypersensitivity reactions. J. Invest. Dermatol. 1994, 102, 934-937.

70. Kuriyama, K.; Shimizu, T.; Horiguchi, T.; Watabe, M.; Abe, Y. Vitamin E ointment at high dose levels suppresses contact dermatitis in rats by stabilizing keratinocytes. Inflamm. Res. 2002, 51, 483-489.

71. Schäfer, U.F.; Loth, H. An ex vivo model for the study of drug penetration into human skin. Pharm. Res. 1996, 13, 336-341. 
72. Haag, S.F.; Bechtel, A.; Darvin, M.E.; Klein, F.; Groth, N.; Schafer-Korting, M.; Bittl, R.; Lademann, J.; Sterry, W.; Meinke, M.C. Comparative study of carotenoids, catalase and radical formation in human and animal skin. Skin Pharmacol. Physiol. 2010, 23, 306-312.

73. Jung, K.; Seifert, M.; Herrling, T.; Fuchs, J. UV-generated free radicals (FR) in skin: Their prevention by sunscreens and their induction by self-tanning agents. Spectrochim. Acta A Mol. Biomol. Spectrosc. 2008, 69, 1423-1428.

74. Sullivan, T.P.; Eaglstein, W.H.; Davis, S.C.; Mertz, P. The pig as a model for human wound healing. Wound Repair Regen 2001, 9, 66-76.

75. Regensburger, J.; Knak, A.; Maisch, T.; Landthaler, M.; Baumler, W. Fatty acids and vitamins generate singlet oxygen under UVB irradiation. Exp. Dermatol. 2012, 21, 135-139.

(C) 2015 by the authors; licensee MDPI, Basel, Switzerland. This article is an open access article distributed under the terms and conditions of the Creative Commons Attribution license (http://creativecommons.org/licenses/by/4.0/). 